\title{
How I would like to foresee the future of theoretic fuzzy logic
}

\author{
Enric Trillas* \\ European Centre for Soft Computing, Mieres (Asturias), Spain
}

\begin{abstract}
Departing from how the speaker became involved, and by what he did work with fuzzy sets and fuzzy logic, an (obviously subjective) look at their future will be discussed. Namely, by arguing on some aspects such research could follow to not only being a theoretic ground for Zadeh's Computing with Words, but also for going towards a new experimental science dealing with the imprecision and non-random uncertainty permeating language and reasoning, as well as with the open question of mathematically modeling ambiguity. That is, to become a new science that, like physics does, can combine careful observations with controlled experiments and suitable mathematical models.
\end{abstract}

Keywords: Fuzzy logic, past, present, future.

\section{INTRODUCTION}

In the XX Century's mid seventies, when I started to study Fuzzy Logic, and in comparison with the today existing large amount of them, there were few papers on it. My fuzzy horizon was, perhaps, clearer and less messy than that of a today's beginner, and I always tended to ask myself for a main final goal, even if it seemed to me not fully affordable.

I would like to recall the papers that, initially, were influential in my own way towards fuzziness. They were the one by Karl Menger on hazy sets [1], the one by Max Black on the consistency profile of a word [2], and the one by Bertrand Russell on vagueness [3]. It was due to these papers that when, by chance and in the fall of 1975, I met the 1965 Zadeh's paper on fuzzy sets [4], I could appreciate that it was not in a usual logico-mathematical direction, but a first trial for considering imprecise words with the help of Mathematical Analysis; that is, by introducing continuity in the analysis of language. Something that latter on I knew it was suggested before by the late John von Neumann [5], and for representing not rigid, not all-or-none, concepts; those that are named by imprecise predicates.

I arrived at Zadeh's paper through a pretty curious way. In the summer of 1975, and one year

\footnotetext{
*Dedicated to those colleagues who worked with me on what I liked to work.
}

after having been appointed to my first chair, it was by an interview with Arnold Kaufmann in a French newspaper that a then young friend supplied to me, that I knew his book on 'Ensembles flous' [6]. In that time, the last years of Franco's totalitarian regime, most Spaniards were reading and hearing non-Spanish media, for getting liable information.

Since Menger did introduce hazy sets in a paper in French, and in which he called them 'ensembles flous', I bought Kaufmann's book thinking that fuzzy sets were related with hazy sets. Even if, in a first moment, I was disappointed by reading the Kaufmann's book that seemed to me a scarcely mathematical one, and not related with probability, some of the examples in it called upon my attention and I decided to look at the paper where fuzzy sets were originally introduced. This decision was one of the most important in my scientific life since I immediately appreciate that fuzzy sets were mathematical representations of imprecise words without more relationship with hazy sets than the fact that these are but a particular case of fuzzy sets. I will always remain in debt with Arnold Kaufmann by the opportunity his book opened to me.

What for me constituted a kind of 'revelation' was the feeling that for the first time after what, at least from Frege, was left aside by logicians, and that Russell placed in this world and not in a celestial one, was taken into account by means of a mathematical model with functions remembering both Black's consistency profiles, and Russell's 'fuzzy photos'. A loop was suddenly closed in my mind where the new possibility of mathematically modeling language without stopping at Discrete Mathematics, was like a seed that in a short period of time flourished like a nice tree. Zadeh's ideas seemed to me the first point for a new Kuhnian revolution in science, and, since then, the idea of mathematically modeling natural language and commonsense reasoning never abandoned me. I must confess that having received a university mathematical education under a strict 'bourbakism', and having reached a chair for life, the possibility of devoting my research's efforts towards a new field that I saw as clearly close to the realities of language and ordinary reasoning, as well as one in which a lot of mathematical work was open to 
be done, did enthusiasm me. An enthusiasm that accompanied me in the last forty years.

It was a time in which very few great scientists believed that Fuzzy Sets were something to be taken into account. I remember, again in the last seventies, that my good friend Bert Schweizer, a brave mathematician, asked me very seriously if I was thinking of solving the problems of language with just the most simple mathematics of the unit interval. Nevertheless, and from the very beginning I counted with the views of two open minded great geometers of the XX Century, Karl Menger in Chicago, and Luis A. Santaló in Buenos Aires, that, although with some reservations by the first, were not refusing fuzzy sets and kept their eyes open to the new field. I also remember that, early in 1976 , and after regretting Zadeh's ignorance on his work, Menger said to me: 'Provided Abraham Wald did not pass away, fuzzy sets will be today a new rich branch of mathematics'. By its part, Santaló always believed in the importance of fuzzy logic for Computer Science, and I can still remember his interest on the subject of T-Indistinguishabilities on which I was thinking of in the early eighties. It were the open views of these great mathematicians that aimed me to challenge, at my own risk of course, the adverse view then kept by most important professors in the world's 'establishment' of mathematics. In a not too long period of time, it meant for me to change from a chair in a Mathematics Department, to one in a Computer Science one. Today, and even if with Horacio's Odes, 'Nihil est ab omni parte beatum', I cannot consider it to my regret.

\section{MY FIRST TIME IN FUZZY LOGIC}

\section{1 .}

Up to some extent, each researcher shows a kind of slavery of what he/she learnt. It is for this reason that, at the beginning, I was only able to imagine fuzzy sets as the probability that the elements in a crisp universe of discourse belong to a crisp subset of it as, more or less, was Menger's idea on hazy sets. My graduate formation took place in a department of Probability and Statistics, where my Ph.D. Dissertation was on Menger's Probabilistic Metrics inside the general view of the Generalized Metric Spaces of which ordinary metrics, Fréchet écarts, Blumenthal's Boolean Metrics, as well as Menger Probabilistic Metrics, are particular cases by also including distances valued in ordered semigroups. I was working on these subjects up to the mid eighties of the last Century. In that time and from the mid sixties, thanks to the papers by Bert Schweizer and Abe Sklar [7], I could knew both t-norms and t-conorms as ordered dual semigroups in the unit interval, and I learnt to pose and solve problems with functional equations thanks to the book by Janos Aczél [8]. I was also familiar with
Lattice Theory and, in particular with Boolean algebras and Orthomodular lattices that I learnt in the book by Garrett Birkhoff [9] and, in relation with probability, in those by Georges Bodiou [10] and Demetrios A. Kappos [11], as well as in some papers by Gary M. Hardegree for what concerns the rule of Modus Ponens in Orhomodular lattices [12]. It was because of those studies, that I have had the nice opportunity of applying all that to Fuzzy Logic.

By my readings on the wide work of Karl Menger, I was, and I am, both acquainted and passionate by the two blades of the famous methodological principle called the Ockham's Razor: The original Ockham's of XIV Century, 'not introducing more entities than those strictly necessary', and the Menger's addenda of XX Century, 'not adding less than those that can conduct to reach significant results' [13]. Trying to pose and work in problems by using a minimal number of suppositions allowing to see the problem from its roots is a research's strategy I always employed in my work. It produces me a psychological sensation of peace and beautifulness in searching what is strictly necessary for posing the problem, and what is sufficient for being able to say something on it, besides leaving room enough for constraining the suppositions up to reach some particular results. In short, by complementing Ockham's Razor with the so-called 'Plato's lifeboat' [45], that a theory should be comprehensive enough to 'save the phenomena', in my case those in language and reasoning showing a non-stationary and open-texture character [46].

\section{2 .}

Once I decided to work within the new fuzzy sets theory, I searched for a subject to which contribute, and I did find the then recent 1972 paper on fuzzy entropies by Aldo de Luca and Settimo Termini [14], that initially called upon my attention not only and just by its title, 'A definition of a non-probabilistic entropy in the setting of fuzzy sets theory', but because the authors were working at a Institute of Cybernetics. In fact, Cybernetics was an almost ended subject, but on which those people in my generation heard a lot, and I did know the work done by Norbert Wiener trough a lecture he delivered in Barcelona and, specially, by his book in which he introduced the name Cybernetics [15]. I have had some idea to approach fuzzy sets by an analogical view in front of a digital one for crisp sets, something that actually I never did continue even if, in the first eighties, I published some work on ideas close to a then new type of analogical computer [42]. The reading of de Luca-Termini's paper introduced me in the new kingdom of measuring fuzziness, a concept that, new for me, I immediately linked with that of measuring the booleanity, or crispness, of a fuzzy set. In the next years I published some papers on the entropy's subject [16] and, as a nice conse- 
quence, introduced me personally with both Aldo and, specially, Settimo with whom I started a close friendship solidly continued for more than thirty five years. All this helped me to consider fuzziness as a measurable restriction of the philosophical wide concept of vagueness and, later on, as the basis for seeing fuzzy sets as just measures of the qualitative meaning of either imprecise, or precise, but not ambiguous, predicates. In addition, it is thanks to Settimo Termini that I became interested, and did some reflections, on some philosophical aspects related with fuzzy sets and fuzzy logic.

\section{3.}

While working on finding models for fuzzy entropies, from very general forms to those specifically coming from either distances, or from a T-indistinguishability between the fuzzy set and its pseudo-complement, I became interested in the representation of the typical connectives 'and', 'or', 'not', and 'if/then'. It happened thanks to several papers among which I would like to quote those by Bellman and Giertz [17], Lowen [18], and Bandler and Kohout [19]. My first contribution to this subject of 'fuzzy connectives' was the characterization, in 1979, of the functionally expressible strong negations [20] I reached thanks to solving a simple functional equation under the axioms posed by Lowen, and that some time latter was continued by a general characterization of involutions by my good friend Sergeí Ovchinnikov [21] with whom, in that time, I maintained continuous conversations. This last work, nevertheless, did not clarify that in fuzzy logic the classical case should be preserved and, consequently, it contains some confusion between antonyms and negation.

Just concerning antonyms, actually important in fuzzy logic for at least constructing linguistic variables, it was a problem that Zadeh posed to me in 1981, and on which then we were not in agreement. It took me around twenty years to well focalize such problem, and it was thanks to the simple example done by the predicate 'around four' in the interval $[0,10]$ where, following Zadeh's idea, the reached antonym is 'around six' that, clearly, is not the expected antonym 'far from four'. This example did show me that each antonym requires to be defined by means of a particular symmetry associated to the predicate and in the universe of discourse, and that, although and if only in a part, the old Ovchinnikov's paper did contain, in fact, the solution. With such solution [22], with which it is easy to find 'far from four' as the expected antonym of 'around four', it is also possible to find the antonyms of predicates that are not in the language, as it is for example, the antonyms of the precise 'less than four', or of the imprecise 'probable'. This line of thought, conducted to see that negation and antonym are not independent concepts, that once fixed a symmetry for the antonym, the negation for the complement should preserve an inequality, and reciprocally. We called such an inequality, showing that the negation is an upper limit (usually inaccessible) of the antonyms, of 'consistency' between negation and antonym [23].

This kind of things should be known by those designing a fuzzy system; not to take them into account can easily conduct to reach a design in fuzzy terms of a problem different of the current one. It is because of this that fuzzy practitioners need to have a theoretic knowledge equivalent to, at least, a first course in fuzzy logic and whose mathematical armamentarium should be intelligently used. I think that the lack of such a course is what causes that many applications are usually anchored only to the triplet (min, max, 1-id), and with the antonym components of the linguistic variable not consistent with their negation. I deeply regret it.

If antonyms were unsuccessfully rounding in my head for more than twenty years synonyms, and earlier than 1984, immediately directed me towards the idea of fuzzy indistinguishability [24]. In fact, synonymy shows the phenomenon consisting in the breaking of its chains, that is, after a few number of synonyms of a given linguistic term, it is reached a final term that is not synonymous with the first. It reminded me the problem that, posed by Poincaré, is called that of the 'physical continuum', and shortened by 'A $=\mathrm{B} \& \mathrm{~B}=\mathrm{C}$, but $\mathrm{A} \neq \mathrm{C}$ '.

Let me stop for a while to remember the only time in forty years I saw Zadeh really angry with someone. It was in 1980 and at a seminar in Berkeley, where a young student was presenting I don't remember what, and he said that the first to pose something was Henri Poincaré. Gesticulating like an important professor, he wrote in the blackboard 'Pointcarre'. Suddenly, Zadeh changed his face, jump from his seat to the blackboard, delete the letters ' $t$ ' and one ' $r$ ', placed an accent on the 'e', and shut-off: 'You must check how names are written!'.

Before the introduction of fuzzy indistinguishabilities, and thanks to my former acquaintance with t-norms and t-conorms that came from my work in Probabilistic Metric Spaces, I presented in 1981, at a seminar in Berkeley, what then it was a new view on the logical connectives for fuzzy sets theory. Before that presentation, I published in 1980, and with Alsina and Valverde, a paper that is almost unknown [25]. Further we jointly wrote a new version that, published in an international journal [26], I think is the first theoretic study on non-distributive representations of the connectives 'and', and 'or', and meant a wide use of t-norms and t-conorms and, later on, of its generalizations. 
After many years in which my only way of considering the representation of imprecise rules, was as 'logical implications', and worried by an example in a Zadeh's paper concerning his Generalized Modus Ponens, in which when the input was just the rule's antecedent the output did not coincide with the rule's consequent, I tried to search for how Modus Ponens was formalized in algebraic logic. I remembered several papers by Gary M. Hardegree, posing the problem for the logic of Quantum Physics, and that I used to pose it in Fuzzy Logic [27] with the restriction of preserving the classical case, a preservation that is necessary since, in the representation of rules not only appear imprecise concepts, and nobody can doubt of how things run in these cases. In this way started a series of many papers that arrived at this Century, and that ended with the formalization of several schemes of classical reasoning in fuzzy logic like they are, for instance, the ones of disjunctive reasoning, resolution, reduction at absurd, etc [28]. In the mean time, the idea of fuzzifying the notion of Tarki's consequence operator both in a crisp and in a graded form, also occupied my brain and, in a certain form, moved me ahead and towards the formalization and the classification of conjectures [29]. A crucial moment towards it was, after 1994, the controversy around the polemical and curiously awarded paper by Charles Elkan [30].

\section{A POINT OF INFLEXION}

Let me shortly reflect on the polemics between Alsina and me against Elkan. In my view the controversy came out from confusing the respective goals of logic and fuzzy logic, something of which I was finally aware during the previous thinking on Elkan's proof before writing our two first papers [31, 32] on Elkan's theorem, whose proof is absolutely bizarre. Only a non-mathematical mind can try to prove the theorem as Elkan did, and only a person ignorant of what theoretical fuzzy logic contained in 1993 could use it with the purpose of posing an apparent paradox.

In the first place, the representation of a rule by means of a direct translation of the classical, Boolean, material implication, makes clear what I just said on a confusion between goals; for instance, by using the conjunctive representation usual in fuzzy control, instead of a material one, Elkan's conclusion obviously fails. What it can be the negation of an antecedent is not always known and, less again, it is always in the corresponding knowledge-base; this often makes unfeasible the use of material conditionals. Even more, in the setting of Ortholattices and also in that of De Morgan algebras, the material conditional forces the lattice to be a Boolean algebra, and the structure in which Elkan develops its paper is just a De Morgan algebra. In addition, the identification of 'not a or b' with 'b' and 'not a or not b', essential in Elkan's proof, is just a Boolean property. What Elkan asserted only happens with Boolean elements that, in the De Morgan algebra of the unit interval, just means the set $\{0,1\}$. Hence, Elkan actually proved nothing, he tried to use a big caliber's artillery to chase flies.

By the way, it should be remarked that all this was more or less coincidental with Zadeh's new idea on Computing with Words [33] that represents a return to the foundational idea for fuzzy sets and fuzzy logic, its use for dealing with the imprecise language that is pervasive in ordinary reasoning where some laws, like the commutative for conjunction, or the De Morgan laws of duality, are not always valid. It was, for instance, exemplified in a paper with Moraga and Alsina in which the old index of Balthazard for adding impairments was formalized in a fuzzy setting [34], and also in another paper in which Alsina and me did study when does the von Neumann's law of perfect repartition hold with fuzzy sets [35], and finding that what cannot be supposed at all is duality. There are a lot of lattice's laws that cannot be presumed in natural language and, hence, they are not only superfluous but able to conduct to conclusions only valid in other domains. Fuzzy logic cannot be seen with the eyes of logic; their agendas are different. To afford fuzzy logic as if it were an abstract domain is not realistic, and can conduct towards a kind of metaphysics. To study some mathematical properties of, for instance, implications is obviously a legitimate work, but it cannot be qualified as something proper of fuzzy logic except if it directly tries to pose or solve a problem of it. In a mathematical study of language and ordinary reasoning, the use of words, the dynamical linguistic experience, plays a role that often surpasses logic.

In addition, for using the De Morgan lattice the connectives min and max generate in the unit interval, it is needed to count with a big amount of information that almost never can be obtained; for instance, to assume that the representation of 'and' is the lattice's minimum it is needed, at least in principle, to know all that is below each of the two arguments, and also that the minimum is the greatest element of all that. Often, such use is just a hypothesis that, to be accepted, needs of some testing, apart from the fact that min does not mix the values of the arguments, is not interactive. It is for this last reason that, in the Takagi-Sugeno method it is preferred the product instead of the minimum used in the Mamdani's method.

It is for reasons of such type, that carefully designing in fuzzy terms a linguistically described sys- 
tem is essential. Fuzzy logic is not only a matter of degree, as Zadeh often says, but its praxis is also a matter of design. And, in design, the meaning of the involved linguistic terms, that depend on the context in which they are used and on the purpose for its use, is basic. Fuzzy problems are essentially semantic, and the corresponding syntax between fuzzy sets not only is not universal, but dictated by 'meaning'.

\section{MEANING}

Fuzzy sets are always seen as their membership functions, usually valued in the unit interval, and by adding that they represent the linguistic label designating the fuzzy set. Then, the first question is what this means and, consequently, how to well design such membership function, that is, from which information it can be designed, and what it actually represents since it is well known that there is not a one to one correspondence between linguistic labels and membership functions.

Provided the linguistic label, or predicate, is precise, the representation, by the preservation principle, is just the characteristic function of the unique crisp set it specifies. But, how can the membership function of an imprecise predicate be specified? That is, how can the meaning of some imprecise predicate $\mathrm{P}$ in a universe $\mathrm{X}$ be described? Of course, the use of such a predicate cannot be 'defined' à la 'if and only if' like it is the case of precise predicates, and, perhaps and as it happens many times, it only can be 'described' by means of some rules on its use, obviously also containing some imprecise terms. The big question is then: What does actually the membership function of an imprecise predicate in a universe of discourse represent?

In front of the predicate $\mathrm{P}=$ odd, of two natural numbers it can never be said that one of them is 'less odd' than the other, nor that one of them is 'very odd', except if previously specifying what 'less odd' and 'very odd' mean beyond odd. But of $\mathrm{P}=$ bitter, for instance, it is in principle possible and thanks to the gustative human perception; we often say that this is less bitter than that, and that a drink is very bitter. With precise predicates, and by only attending to what they mean, there is only the binary relation ' $\mathrm{a}$ is equally $\mathrm{P}$ than $\mathrm{y}$ ', but with the imprecise ones it is possible to recognize that ' $a$ is less $\mathrm{P}$ than b', or, equivalently, that ' $\mathrm{b}$ is more $\mathrm{P}$ than a'. Without such recognition [36], it is impossible to state that $\mathrm{P}$ is imprecise in $\mathrm{X}$.

In the precise case, the relation 'equally $\mathrm{P}$ than' is just an equivalence allowing to classify the universe in the two crisp sets specified by $\mathrm{P}$, those that 'are P', and those that 'are not P'. In the imprecise case, this relation is just the intersection of the two inverse relations defined by ' $\mathrm{a}$ is less $\mathrm{P}$ than b', and ' $\mathrm{b}$ is less $\mathrm{P}$ than a', and that only is an equivalence depending on the properties the relation 'less than' can enjoy. Of course, this last relation is empirical and perceptively known by how $\mathrm{P}$ is used in $\mathrm{X}$, and often it can be linguistically expressed by words through, for instance, some linguistic rules. Hence, a predicate for which such a relation can be expressed, is imprecise in the universe that, then, results 'organized' in the very simple form given by the graph constituted by it and the relation. It corresponds with the intuitive idea that a first goal of intelligent speaking consists in introducing some 'order', or 'organization', or 'structure', in the universe of the objects on which something is said. The contrary of a pointless talk.

Such graph can be Christianized as the qualitative meaning of $\mathrm{P}$ in $\mathrm{X}$, and then it can be said that $\mathrm{P}$ is measurable in $\mathrm{X}$. By the contrary, when such crisp graph cannot be, at least partially, specified, it can be said that $\mathrm{P}$ is metaphysical or meaningless in $\mathrm{X}$; a meaningless $\mathrm{P}$ has an empty relation 'less than'. In my view, this clarifies what the members of the Vienna Circle tried to state in the twenties of last Century in their fight against metaphysics in the philosophy of Science [37].

Once a graph is known, and there are isolated those of their objects that are either maximals, or minimals, and provided they exist, a general measure of the qualitative meaning of $\mathrm{P}$ in $\mathrm{X}$ can be introduced as a mapping that assigns a number in the unit interval to any element in the universe $\mathrm{X}$, and such that is non-decreasing respect to the relation 'less than', assigns the values 1 to the maximals, 0 to the minimals, and no additive law is supposed. Obviously, and in the same way it happens with probabilities, this definition cannot specify a single measure except if the predicate is precise and, hence, it has only two classes whose values will be 0 or 1 . Either more information, or some reasonable hypotheses, on the context and purpose on which $\mathrm{P}$ is used, can be sufficient to specify a measure, and that, once specified and jointly with the graph, will represent a meaning of $\mathrm{P}$ in $\mathrm{X}$. For instance, in some cases and for some purpose, a reasonable well known hypothesis is the trapezoidal character of the measure. All that is very close to what happened with measures like those of length, surface, volume, etc., and shows that the subjects to be represented by means of fuzzy sets are those linguistic labels that are measurable in some universe of discourse, that is, can be scientifically considered in it. Each meaning is, thus, specified by a triplet, or quantity, (X, relation 'less than', measure), and each measure is just a contextual membership function of the fuzzy set labeled $\mathrm{P}$. 
If, by one side, it can be roughly said that 'people only eat what weights', by the other it is important to recall what Lord Kelvin's wrote [43], and that can be shortened by 'If you cannot measure it, it is not science'. Once membership functions are seen as measures of the qualitative meaning of a predicate, and since a designer often cannot capture it completely, it should be noticed that in most practical cases the membership functions are but approximations to the measure, and, consequently, it lacks to prove a theorem similar to that in fuzzy control under which the difference between the function directly defining a dynamical system, and its nominal, fuzzy-defuzzified function coming from CRI, is very small. By another side, with this idea of meaning as a quantity it is not difficult to look at the, historically dangerous, concept of 'truth' as a non-metaphysical one.

This view of fuzzy sets goes far from logic, and is according with the known fact that each fuzzy set is defined, at each context, by a membership function, but it should be complemented by specifying a suitable structure in the set of all fuzzy sets in X, and once endowed with a graded ordering instead of the usual and too crisp pointwise ordering. Up to this moment, the only that I did is the definition of a very general algebra of fuzzy sets in two directions, the crisp one with the pointwise ordering, and the graded one with a fuzzy ordering, that is, with a fuzzy relation such that at the degree one it coincides with the pointwise order [38]. These algebras are endowed with two binary operations able to represent the linguistic 'and', and 'or', without too many laws like, for instance, the character of being functionally expressible, the commutative law, the associative and those of duality, but are easy to particularize in the Standard algebras with t-norms and t-conorms. Analogously, the negation is neither functionally expressible, nor strong.

I called these algebras 'Basic Algebras' of fuzzy sets, and I think they are a wide setting where not all of them, but a good part of the nuances and particularities of language can be modeled by, sometimes, strengthening the laws. No one of them can be, with all fuzzy sets, an Ortholattice and less again a Boolean algebra, and the only that are lattices are those with the typical and very restrictive connectives $\min$ and $\max$ that, with a very week constraint on the negation, are De Morgan-Kleene algebras [39]. Lattices enjoy too many laws for what concerns the representation of natural language and ordinary reasoning.

From all that, there follows a new view that can be summarized by saying that, properly, fuzzy sets theory is neither a mathematical, nor a logical one, even if each basic algebra responds to a general mathematical model that should be restricted at each case for obtaining some wished, or necessary, laws. Since the triplet defining the fuzzy set is nothing else than a quantity, I think that these reflections open the window for seeing fuzzy sets theory far from logic, and close to an experimental science. Let me present three examples that can help to clarify what I would like to say and that refers the opposites, or antonyms, of a predicate, the pervasive presence of ambiguity in language, and the conjunction and disjunction of predicates.

a) If the meaning of $\mathrm{P}$ in $\mathrm{X}$ is given by the quantity expressed by a graph and a measure, then the first question that should be answered is if an existing antonym aP, is measurable and how its measures can be obtained from those of $\mathrm{P}$. This is not at all difficult to reach, and if no doubt the involutive law $\mathrm{a}(\mathrm{aP})=\mathrm{P} \Leftrightarrow \mathrm{a}^{2}=$ $\operatorname{id}_{X}$ can be accepted, it lacks to know what happens with different antonyms like, say, $a_{1}$ and $\mathrm{a}_{2}$, that is, with the compositions $\mathrm{a}_{1} \circ \mathrm{a}_{2}$. For instance, if $\mathrm{P}=$ sweet, $\mathrm{a}_{1} \mathrm{P}=$ bitter, $\mathrm{a}_{2} \mathrm{P}=$ salty, is it $\mathrm{a}_{1}\left(\mathrm{a}_{2} \mathrm{P}\right)=\mathrm{a}_{1}($ salty $)=$ sweet?

b) Another topic for whose study there is a lack of practical knowledge is that of ambiguity. Notice that to manage with a predicate showing several meanings in the same context, it seems necessary to deal with the several quantities reflecting each one of them, something that at least requires of some previous observation in language. As far as I know, ambiguity did not receive, in itself, formal attention like it happened, for instance, with imprecision and uncertainty; linguistic ambiguity remains open to a scientific scrutiny.

c) If $\mathrm{P}$ and $\mathrm{Q}$ are measurable on $\mathrm{X}$, then a first question is to study if the predicates ' $\mathrm{P}$ and $\mathrm{Q}$ ', and ' $\mathrm{P}$ or $\mathrm{Q}$ ', are also measurable on $\mathrm{X}$, as well as to find general enough forms for their membership functions. For the conjunction ' $\mathrm{P}$ and $\mathrm{Q}$ ' is easy to recognize that their relation 'less $\mathrm{P}$ and $\mathrm{Q}^{\prime}$ is just the intersection of the respective relations 'less $\mathrm{P}$ ' and 'less Q', but for the predicate ' $\mathrm{P}$ or $\mathrm{Q}$ ' the only that can be said is that the relation 'less $\mathrm{P}$ or $\mathrm{Q}$ ' includes the union of the respective relations and, hence, it is not empty. Hence both predicates ' $\mathrm{P}$ and Q', and ' $\mathrm{P}$ or $\mathrm{Q}$ ' are measurable, although I only have been able to reach the respective forms of their membership functions under some particular suppositions.

To deeply study such kind of questions it is needed to count with some previous and systematic work in language, that is, some observation and perhaps controlled experimentation in it. Their relevance follows from the fact that, at the end, 
mathematically modeling language can be considered as a part of the so-called 'Gordian Knot' of Artificial Intelligence.

\section{Remarks}

1) What has been said refers to predicates, that is, to words naming properties recognizable in the elements of the universe of discourse, but not to true proper names [44]. Proper names just serve to specify a single individual, not only characterized by some physical characteristics, but also by a profession, its belonging to a family, etc. In this sense, and for instance, the only meaning attributable to 'Caesar' can be seen as just the singleton $\{$ Caesar , a subset of all characters recorded in History.

2) According to Russell [3] and in most cases, the concept of meaning also touches Psychology since, due to the perceptive character of the relation 'less than', it is endowed with some subjectivity.

\section{A MODEL FOR COMMONSENSE REASONING}

Ordinary, everyday, or commonsense reasoning is not fully deductive and less again in the same form typical of a mathematical proof. I doubt that the ways of deductive reasoning can even approach the $15 \%$, and, hence, the $85 \%$ rest of non-deductive forms of reasoning are very important. Provided 'Computing with Words' will take care of ordinary reasoning, it should consider them.

Even a mathematician, and before trying to formally conduct a proof, reasons like people do by conjecturing something, and only after 'seeing' what he/she is trying to look for, and often by self-proposing some examples, tries to prove what conjectures. All scientists must work by conjecturing on what is calling up his/her interest, and each branch of science has its own standards for accepting or refusing the different ways to prove something and, with it, certifying its knowledge. Of course, in commonsense reasoning such standards are weaker than in the hard sciences but, nevertheless, in all cases conjecturing and refuting are the main activities of reasoning, often assisted by analogy with former reasoning. Let me stop for a while and tell what, perhaps, is nothing else than a curiosity.

Catalan, but neither Spanish, nor French, nor Italian, nor German, nor English, nor Portuguese, etc., distinguishes between the two words 'parlar' (to speak) and 'enraonar' (something like speaking with reasons). As far as I know, only Aranese, a very old language in the Pyrenaic Aran's Valley, has an equivalent word, 'arrasoar', with a clear
Basque root even if Basque language has not it. It seems that from very old some cultures tried to clearly distinguish between just speaking and speaking with reasons, something in between speaking and arguing.

If cleaver minds always tended to escape from pointless, meaningless talking, it was Greek geometry what did show how to safely reason in such a way to identify possible mistakes and trying to correct them; in sum, of reasoning without dangerous jumps. May be it was the fame Euclid's 'Elements' acquired, what conducted to identify 'correct reasoning' with deduction; of course, not a bad identification when there is a formal setting in which deduction can be, step-by-step, made explicit. Notwithstanding, most of human debates, conversations, controversies, searches, etc., are not at all deductive like geometry is, and, for instance, as Popper did show [40], also science is done by conjecturing and refuting. Most of our scientific knowledge consists but of provisory conjectures both well grounded in the observable reality, and not contradictory between them. By its side, ordinary reasoning often uses metaphors whose danger lies in the, let's say, 'distance' with what is currently commented and that were originated in a different universe of discourse and, probably, with a different purpose. The layman's 'I deduce that', is not equivalent to the mathematician's 'I deduced that', although both concepts have something in common, and with the second having more properties than the first; the kind of deduction it refers to is more restrictive and is conducted without jumps, as well as it allows to be exactly repeated by other mathematician.

Hence, and towards starting with a formalization of ordinary reasoning [41], it seems preferable to do it by taking as a tool the layman's, or informal, deduction and begin to ask for those laws or properties it shares with formal deduction. The word 'preferable' just refers here to not adding those characteristics of formal deduction that are not clearly present in the informal one as it is, for instance, that between the conclusions of a formal deduction, the so-called logical consequences of the premises, there cannot be contradictory pairs of them, and less again self-contradictory ones. Hence, the consequences of an 'informal' deduction cannot be taken as safe information, or premises to further deduce from them, as it is done in formal deduction; that is, the Tarki's law of closure has no sense in informal deduction. Nevertheless, what nobody will refuse is the monotonic law, stating that when the number of premises increases, the number of consequences will not decrease. In any case, ordinary deduction is seen by educated minds as the safest way of conducting ordinary reasoning. 
With that, a refutation can be defined as a conclusion whose negation follows by informal deduction from the résumé of the premises (by calling résumé the conjunction of them), and, thus, a conjecture is a conclusion that is not a refutation, that is, whose negation cannot follow by informal deduction from the premises. Obviously, when a conclusion follows formally from the premises, it keeps the character of refutation or conjecture, respectively. Hence, given a set of premises, the only elements that can be found are conjectures and refutations, and for a suitable formalization it lacks to represent in some formal setting what is intended by 'deductively follows'.

Only avoiding ambiguity, the former 'Basic Algebras' seem to a suitable framework for it, by just taking the pointwise ordering between fuzzy sets as the 'follow up' relation, or the graded one as 'follow up to some degree'. In this framework there is not the big amount of laws usually presumed in classical logic, and, hence, what can be posed enjoys a great generality. It is, of course, a week algebraic approach.

In this setting, conjectures can be easily classified as those that follow from the résumé, those such that the résumé follows from them, and those that are not comparable with the résumé, that is, neither the résumé follows, nor they follow from the résumé. The first are the consequences, the second are the hypotheses, and the third are the speculations that, at its turn, can be classified in two subclasses, those that the résumé follows from its negation and those that the résumé is also not comparable with its negation. Notice that consequences follow forwards from the résumé, hypothesis backwards from it, the first kind of speculations backwards from the negation of the résumé, but and notwithstanding the second type of speculations cannot be reached neither forwards, nor backwards, nor from the résumé, nor from its negation. For reaching these last speculations, no deductive path from the premises' résumé exist, they constitute the true inductive part of reasoning [40]; they are actually 'creative' speculations, the root of the famous 'Eureka!' of Archimede.

With respect to monotony, conjectures, hypotheses and the first type speculations are anti-monotonic, refutations and consequences are monotonic, but the second type speculations are properly non-monotonic since they are neither monotonic, nor ant-monotonic. Both its inductive character and non-monotony help to see that the search for these speculations aims at reaching new ideas not hidden in the premises, to enlarge its informational content that, as it is known, cannot be obtained by deduction. Is for that that I called them 'creative speculations'; its study, and the search for computational methods to reach them or heurisdics, is of a paramount importance for Artificial Intelligence.

This model in BA, not only allows to formalize Popper's falsification of hypotheses, but also to see for what the speculations are useful to thinkers: its conjunction with the résumé is a hypothesis, and its disjunction a consequence. Speculation allows both to deploy what is hidden in the premises, and to explain them. Explanation is the general goal of science.

It is just a mathematical model inspired in very general treats of commonsense reasoning, and whose validity only can come from testing what it states against the reality of actual reasoning, that is, with some controlled experiments with which what the model seems to foresee can be either accepted, or falsified as it is, for instance, that creative speculations are used to explain the premises, to find hypotheses of them, as well as that the act of creating something new is done by means of them. Even if I am convinced that no conclusions different from conjectures and refutations can be reached by analogy, the model still lacks of a deep study of the role analogy plays in the search for hypotheses and speculations and, specially, the creative ones.

\section{CONCLUSION}

1. Mathematical models can help to drive research in some direction better than in another, but they are not the 'truth'. Without checking them with the reality they try to model, are nothing else and nothing less than mathematical constructs whose importance lie, in our case, out of fuzzy logic and its judging only corresponds to mathematicians. What corresponds to, for instance, fuzzy logicians, what matters for them, is the usefulness of the models in fuzzy logic, or, better to say, in Computing with Words. Einstein's relativity theory is a very nice one that without its continuous agreement with observed physical phenomena would be out of consideration by physicists, and I have a lot of doubts concerning the interest and usefulness for fuzzy logic of many mathematical papers published in journals that are not properly devoted to mathematics, even if there are some of them showing a great mathematical quality.

What interest to Computing with Words is, as I see it, what is behind natural language and ordinary reasoning, the ground reality from which it represents the data for posing and solving the practical problems it applies to. This dynamic and complex reality should not, or simply cannot, be taken into account from 
only the abstract point of view of mathematics, whose main interests are not directly linked neither with linguistic imprecision, nor linguistic uncertainty, and less again with ambiguity. It is for this reason that I advocate for a kind of 'physics' of language and reasoning and of which some aspects of classical, deviated, non-monotonic, etc., logics are but partial theoretical models. In this view, mathematical modeling is basic. In the Virgil's words of the Georgics, 'Felix qui portuit rerum cognoscere causas'.

2. Fifty years after its inception it is, perhaps, a good moment to reconsider what we did and are still doing. I hope and wish a young generation of theoretical researchers in Computing with Words that would like to affront such reconsideration without refraining the reporting of failed ideas. I would be delighted if a new and more open and sincere type of scientific communication, in both the personal scientific relationships and also in conferences, could appear in a new way for theoretical fuzzy logic.

Up to some extent, I see as something bizarre that in the Century what most people consider is the 'brain's functioning' one, however no experimental researches in both meaning and reasoning are in course in the setting of Computing with Words. At the end language and reasoning are but external manifestations of the natural phenomenon usually called 'thinking' that develops in the brain.

3. Let me end by encouraging young researchers to go along with such a task by not only trying to fit a curriculum to scholarly survive in the Academia, but by looking far in the search of a challenging last goal. I wish them to work with the passionate enthusiasm with which the generations of the sixties, seventies and eighties of last Century did start researching in fuzzy $\operatorname{logic}$.

\section{Acknowledgment}

This paper is partially supported by the 'Foundation for the Advancement of Soft Computing', Mieres (Asturias), Spain.

\section{References}

[1] K. Menger, Ensembles flous et fonctions aléatoires, Comptes Rendues Acad. Sci. Paris, Vol. 232, pp. 2001-2003, 1957.

[2] M. Black, Vagueness: An exercise in logical analysis, Philosophy of Science, Vol. 4/4, pp.
427- 455, 1937.

[3] B. Russell, On Vagueness, Australasian Journal of Psychology and Philosophy, Vol. I, pp. 84-92, 1923.

[4] L.A. Zadeh, Fuzzy Sets, Information and Control, Vol. 8, pp. 338-353, 1965.

[5] J. von Neumann, The General and Logical Theory of Automata, Lecture at the Hixon Symposium , Pasadena, Cal, 1948.

[6] A. Kaufmann, Introduction a la théorie des sous-ensembles flous à l'usage des ingénieurs, Masson, Paris, 1975.

[7] B. Schweizer, A. Sklar, Probabilistic Metric Spaces, Elsevier, New York, 1983.

[8] J. Aczél, Lectures on functional equations and their applications, Academic Press, New York, 1966.

[9] G. Birkhoff, Lattice Theory, AMS Colloquium Publications, New York, 1948.

[10] G. Bodiou, Théorie dialectique des probabilités, Gauthier-Villars, Paris, 1964.

[11] D.A. Kappos, Probability Algebras and Stochastic Spaces, Academic Press, New York, 1969.

[12] G.M. Hardegree, Material Implication in Orthomodular (and Boolean) Lattices, Notre Dame Journal of Formal Logic, Vol. 22/2, pp. 163-182, 1981.

[13] K. Menger, A counterpart of Ockham's razor in pure and applied mathematics, Synthese, Vol. 12, pp. 415-428, 1960

[14] A. de Luca, S. Termini, A definition of a non-probabilistic entropy in the setting of fuzzy sets theory, Information and Control, Vol. 20, pp. 301-312, 1972.

[15] N. Wiener, Cybernetics or Control and Communication in the Animal and the Machine, MIT Press (Cambridge, Mass), 1948.

[16] N. Batle, E. Trillas, Entropy and Fuzzy Integral, Jour. Mathematical Analysis and Applications, Vol. 69/2, pp. 469-474, 1979.

[17] R. Bellman, M. Giertz, On the Analytic Formalism of Fuzzy Sets, Information Sciences, Vo. 5, pp. 149-156, 1973. 
[18] R. Lowen, On fuzzy complements, Information Sciences, Vol. 14/2, pp. 107-113, 1978.

[19] W. Bandler, L. Kohout, Fuzzy power sets and implication operators, Fuzzy Sets and Systems, Vol. 4/1, pp. 13-30, 1980.

[20] E. Trillas, Sobre funciones de negación en la teoría de subconjuntos difusos, Stochastica, Vol. III/1, pp. 47-59, 1979.

[21] S. Ovchinnikov, General negations in fuzzy set theory, Jour. of Mathematical Analysis and Applications, Vol. 92, pp. 234-239, 1983.

[22] E. Trillas, C. Moraga, S. Guadarrama, S. Cubillo, E. Castiñeira, Computing with Antonyms, Forging New Frontiers (Eds. M. Nikravesh et altri), Springer, Berlin, pp. 133-153, 2007.

[23] E. Trillas, S. Termini, C. Moraga, A naïve way of looking at fuzzy sets, Fuzzy Sets and Systems (forthcoming), 2015.

[24] E. Trillas, Ll. Valverde, An Inquiry into Indistinguishability Operators, Aspects of Vagueness (Eds. H.J. Skala, et altri), pp. 231-256, 1984.

[25] C. Alsina, E. Trillas, Ll. Valverde, On nondistributive logical connectives for fuzzy set theory, Busefal, Vol. 3, pp. 18- 21, 1980.

[26] C. Alsina, E. Trillas, Ll. Valverde, On Some Logical Connectives for Fuzzy Set Theory, Jour. Of Mathematical Analysis and Applications, Vol. 93/1, pp. 15-26, 1983.

[27] E. Trillas, Fuzzy Sets: Towards the Scientific Domestication of Imprecision, ECSC's Research Report (available upon request), 2015.

[28] E. Trillas, C. Alsina, E. Renedo, On some classical schemes of reasoning in fuzzy logic, New Mathematics and Natural Computation, Vol. 7/3, pp. 433-451, 2011.

[29] E. Trillas, S. Cubillo, E. Castiñeira, On conjectures in orthocomplemented lattices, Artificial Intelligence, Vol. 117, pp. 255-275, 2000.

[30] Ch. Elkan, The paradoxical success of fuzzy logic, IEEE Expert (August), pp. 698- 703, 1994.

[31] E. Trillas, C. Alsina, Elkan's theoretical argument, reconsidered, International Journal of Approximate Reasoning, Vol. 26/2, pp. 145-162, 2001.

[32] E. Trillas, C. Alsina, Comments to 'Paradoxes of fuzzy logic, revisited', International Journal of
Approximate Reasoning, Vol. 26/3, pp. 157-159, 2001.

[33] L. A. Zadeh, Computing with Words, Principal Concepts and Ideas, Springer, Heidelberg, 2012.

[34] E. Trillas, C. Alsina, C. Moraga, Combining Degrees of Impairment: The case of the Index of Balthazard, Mathware \& Soft Computing, Vol. 10, pp. 23-41, 2003.

[35] E. Trillas, C. Alsina, Standard theories of fuzzy sets with the law $\left(\mu \cdot \sigma^{\prime}\right)^{\prime}=\sigma+\mu^{\prime} \cdot \sigma^{\prime}$, International Journal of Approximate Reasoning, Vol. 37/2, pp. 87-92, 2004.

[36] E. Trillas, On the Genesis of Fuzzy Sets, Agora, Vol. 27/1, pp. 7-33, 2008.

[37] E. Trillas, R. Seising, 2015, On meaning and measuring: A philosophical and historical view, Agora, Vol. 34/2, (forthcoming), 2015.

[38] E. Trillas, A. de Soto, Some Comments on Ordinary Reasoning with Fuzzy Sets, ECSC's Research Report (available upon request), 2015.

[39] E. Trillas, A model for 'crisp reasoning' with fuzzy sets, International Journal of Intelligent Systems, Vol. 27/10, pp. 859-872, 2012.

[40] K.R. Popper, Conjectures and Refutations: The Growth of Scientific Knowledge, Routledge, London, 1963.

[41] E. Trillas, Glimpsing at Guessing, Fuzzy Sets and Systems (forthcoming), 2015.

[42] L. Basañez, N. Batle, J. Grané, G. Ferraté, E. Trillas, A First Mathematical Approach to Sigma-Transform, Journal of Mathematical Analysis and Applications, Vol. 92/1, pp. 224$233,1983$.

[43] W. Thompson (Lord Kelvin), Lecture on 'Electrical Units of Measurement', Popular Lectures and Adresses, Vol.I, McMillan, London, 1889 .

[44] M. Kohl, Bertrand Russell on Vagueness, Australasian Journal of Philosophy, Vol. 47/1, pp. 1-11, 1969.

[45] D. Gernert, Ockham's Razor and Its Improper Use, Journal of Scientific Exploration, Vol. 21/1, pp. 135-140, 2007.

[46] E.L. Rissland, AI and Similarity, IEEE Intelligent Systems, Vol. 21, pp. 39-49, 2006. 